\title{
Theoretical Analysis of Coupled Modified Hindmarsh-rose Model Under Transcranial Magnetic-acoustic Electrical Stimulation
}

\author{
Liang Guo ${ }^{1,2}$, Shuai Zhang ${ }^{1,2,}$, Jiankang $\mathrm{Wu}^{1,2}$, Xinyu Gao ${ }^{1,2}$, Mingkang Zhao ${ }^{1,2}$, Guizhi $\mathrm{Xu}^{1,2}$ \\ ${ }^{1}$ State Key Laboratory of Reliability and Intelligence of Electrical Equipment, Hebei University of \\ Technology, Tianjin 300130, China \\ ${ }^{2}$ Key Laboratory of Bioelectric and Intelligent Health, Hebei University of Technology, \\ Tianjin 300130, China
}

Received: June 21, 2021. Revised: December 12, 2021. Accepted: January 13, 2022. Published: January 15, 2022.

\begin{abstract}
Transcranial magnetic-acoustic electrical stimulation (TMAES) is a new technology with ultrasonic waves and a static magnetic field to generate an electric current in nerve tissues to modulate neuronal firing activities. The existing neuron models only simulate a single neuron, and there are few studies on coupled neurons models about TMAES. Most of the neurons in the cerebral cortex are not isolated but are coupled to each other. It is necessary to study the information transmission of coupled neurons. The types of neuron coupled synapses include electrical synapse and chemical synapse. A neuron model without considering chemical synapses is not comprehensive. Here, we modified the Hindmarsh-Rose (HR) model to simulate the smallest nervous system-two neurons coupled electrical synapses and chemical synapses under TMAES. And the environmental variables describing the synaptic coupling between two neurons and the nonlinearity of the nervous system are also taken into account. The firing behavior of the nervous system can be modulated by changing the intensity or the modulation frequency. The results show that within a certain range of parameters, the discharge frequency of coupled neurons could be increased by altering the modulation frequency, and intensity of stimulation, modulating the excitability of neurons, reducing the response time of chemical postsynaptic neurons, and accelerating the information transferring. Moreover, the discharge frequency of neurons was selective to stimulus parameters. These results demonstrate the possible theoretical regulatory mechanism of the neurons' firing frequency characteristics by TMAES. The study establishes the foundation for large-scale neural network modeling and can be taken as the theoretical basis for TMAES experimental and clinical application.
\end{abstract}

Keywords - Electric field, Firing behaviors, HR model, Transcranial magnetic-acoustic electrical stimulation (TMAES).

\section{INTRODUCTION}

$\mathrm{N}^{\mathrm{c}}$ euromodulation technology, such as transcranial direct current stimulation (tDCS) [1-6], transcranial magnetic stimulation (TMS) [7-10], and transcranial ultrasound stimulation (TUS) [11-14], can treat neuropsychiatric disorders. Neuromodulation technology is increasingly used for the research of information processing in neural networks. However, the electric fields generated by TMS and tDCS are widely distributed, which is unable to locate specific target areas in the brain, with low spatial resolution. Although TUS with high resolution and has clinical potential for treating nervous system diseases, the thermal effect of high ultrasound intensity may cause tissue damage [15-16]. TMAES is emerging as a new type of non-invasive brain stimulation modality, with advantageous characteristics of high spatial resolution and low ultrasound intensity [17]. The modulation of TMAES has been demonstrated in many previous studies. Norton [18] proved that the coupled field generated by propagating ultrasound in a static magnetic field can locally stimulate the biological tissues of the cerebral cortex in the ultrasound focus area. Yuan [19] got the analytical solution for the voltage distribution based on the cable model of intracellular potential under TMAES. We have modified the Izhikevich model [20] of a neuron with the mechanoelectrical effect of TMAES [21]. There are few studies on coupled neuron models with both electrical and chemical synapses. Also, the coupled neuron models about TMAES are still important.

Reliable neuron models provide a method for understanding the potential mechanisms of signal processing in neuroelectric activity under external stimulation. Hodgkin [22] and Huxley 


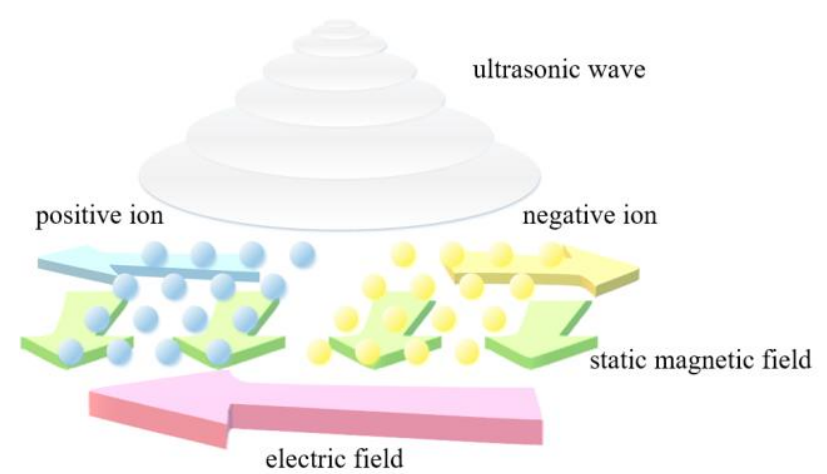

Figure 1. Schematic diagram of TMAES

established a neuron numerical computational model, the Hodgkin-Huxley $(\mathrm{H}-\mathrm{H})$ model, which mainly considers the influence of ion channel current on cell membrane potential. In 1982, Hindmarsh [23] and Rose established a two-dimensional model, the Hindmarsh-Rose (HR) neuron model, which is a simplified H-H model with low complexity and is suitable for the simulation of neurons. In 1984, Hindmarsh [24] and Rose modified the original HR model and added a regulated current variable to make it a three-dimensional model. The HR model is a classic model that describes the firing behavior of neurons. It considers the effects of various ions on action potentials as a whole. It is easier to achieve numerical calculations. It is a common model for analyzing biological neurons and can simulate neuronal electrical activity and frequency characteristics. Some modified HR models have been studied [25-27]. However, there are few studies on neuron model coupled systems about TMAES. The HR model is commonly used with electrical stimulation. It is worth noting that these studies consider only direct current as an external input and it cannot depict the ultrasound. So we modified the HR model to describe the neural firing under TMAES.

Despite the effect of TMAES has been successfully proven in studies, the full mapping that demonstrates the frequency characteristics of the discharge of stimulation parameters synapses especially chemical synapses remains missing. Ma [28] introduced a new field variable into the FitzHugh-Nagumo model [29] to investigate a new neuron model that takes the effects of the electric field into account. Ma [27] added a coupling factor to the HR model that focuses on the information exchange between synapse-coupled neurons under magnetic flow. In order to understand the effects of TMAES on coupled neurons, with the important role of induced electric field effect on neural firing activity considered, the HR model was modified to investigate the firing activities of the coupled neurons under TMAES.

Inspired by the models in [27-28], a new neuron model is proposed by introducing a new field variable to consider the effect of the induced electric field under TMAES. We described the neural discharge activity with TMAES taking into account the changes in membrane capacitance caused by ultrasound. In this paper, we study the effects of magnetic-acoustic electrical stimulation on electrical and chemical synapses based on the modified HR model and carry out theoretical analysis and numerical simulation. We take the smallest nervous system - coupled neurons to conduct research and modify the HR model to describe the mechanism of the induced electric field and ultrasound field of TMAES on neurons, and analyze the effect of TMAES on interacting nerves. Our work provides theoretical evidence on how TMAES regulates the neural activity and supports the predictions as the potential mechanism of memory-enhancing of TMAES.

\section{METHODS}

\section{A. Magnetic-acoustic electrical effect}

TMAES can produce the magnetic-acoustic electrical effect and periodic change of the cell membrane capacitance without causing tissue damage. The proposed model of magnetic-acoustic electrical effect is shown in Figure. 1. Charged ions move in the brain tissue under ultrasound. There is a static magnetic field perpendicular to the ions and ultrasonic waves that subject the charged ions to the Lorentz force. The movement of ions creates an induced electric field.

The particle $q$ oscillates in the tissue at a velocity $v$ by the mechanical effect of ultrasound waves. In the static magnetic field $\boldsymbol{B}$, the Lorentz force $\boldsymbol{F}$ is

$$
\boldsymbol{F}=q \boldsymbol{v} \times \boldsymbol{B} \text {. (1) }
$$

The magnitude of $\boldsymbol{v}$ is described as

$$
v=\frac{P}{\rho c},(2)
$$

where $P$ is the ultrasonic pressure, $\rho$ denotes tissue density and $\boldsymbol{c}$ is the speed of ultrasound in tissue.

Lorentz force is rewritten as

$$
\boldsymbol{F}=q \frac{P}{\rho \boldsymbol{c}} \times \boldsymbol{B} \text {. (3) }
$$

The $\boldsymbol{J}$ is the density of the induced current intensity, besides $\sigma$, is the tissue conductivity, obeys the equation

$$
\boldsymbol{J}=\sigma \frac{\boldsymbol{F}}{q} .
$$

where $\sigma$ is the conductivity of biological tissue.

The classical relation of the Lorentz force $\boldsymbol{F}$ and electric field $\boldsymbol{E}$ can be expressed as

$$
\boldsymbol{E}=\frac{\boldsymbol{F}}{q}=\boldsymbol{v} \times \boldsymbol{B} .
$$

According to the above equation, we obtain the scalar form of $J$ is

$$
J=\sigma \frac{P B}{\rho c} \sin \theta
$$

where $\theta$ is the angle between the magnetic induction intensity of the static magnetic field and the direction of ultrasonic propagation.

Therefore, the main parameters of TMAES are ultrasonic pressure $P$, tissue density $\rho$, magnetic induction intensity $B$, and electrical conductivity $J$. The magnetic-acoustic electrical 
effects on the charged particles in biological tissues can act on brain tissue. The electrical activity of neurons under ultrasound involves the mechanical effect [30-32]. When periodic ultrasound acts on neurons, as the tension generated by the ultrasound increases, the cell membrane deforms, the neuron membrane capacitance is changed, thereby changing the membrane potential and affecting the neuron discharge. The average membrane capacitance is time-dependent. This primarily is attributed to current changes induced by the change of capacitance. Under the action of ultrasound with frequency $f$, the periodic change of the cell membrane capacitance $C_{m}$ per unit area can be approximately derived by [21][31]

$$
\frac{d C_{m}}{d t}=(2 \pi f) C_{a m p} \cos (2 \pi f t),(7)
$$

which describes the electromechanical effect, when $f=690 \mathrm{kHz}$, the membrane capacitance peak value $C_{a m p}$ is 0.8 , and when $t=0$, $C_{m}=C_{m 0}=1.0$, represents the cell membrane capacitance in the resting state [32]. $f$ is ultrasound frequency.

\section{B. Modified Hindmarsh-Rose Model}

The original HR model contains the external current $I_{\text {ext }}$. When the classical HR model is used in the simulation under TMAES, the effect of the induced electric field will be ignored. The neuron model under induced electric field $\boldsymbol{E}_{\text {ext }}$ has been the key factor of the nerve regulation effect of TMAES. We conduct $\boldsymbol{E}_{\text {ext }}$ on the modified HR model to simulate electric field and analyze the biological effects caused by TMAES. The modified HR model is described by the differential equations below:

$$
\left\{\begin{aligned}
C_{m} \frac{d x}{d t} & =y-x^{3}+3 x^{2}-Z+I_{e x t} \\
\frac{d y}{d t} & =1-5 x^{2}-y+r E \\
\frac{d z}{d t} & =0.006[4(x+1.56)-Z](8) \\
\frac{d E}{d t} & =k y+\boldsymbol{E}_{e x t} \\
\frac{d C_{m}}{d t} & =(2 \pi f) C_{a m p} \cos (2 \pi f t)
\end{aligned}\right.
$$

where $x, y, \mathrm{z}$ are the membrane potential, slow, and adaption current, respectively. $I_{e x t}$ is the imposed current and $E$ is the electric field around the neuron, $r$ and $k$ are the inherent parameters of biological tissues, $\boldsymbol{E}_{\text {ext }}$ is the external electric field, which represents the TMAES. $\boldsymbol{E}_{\text {ext }}$ can generate current $I_{\text {ext }}$, and $I_{\text {ext }}=\sigma \boldsymbol{E}_{\text {ext }}$, where $\sigma$ was 0.276 [33].

\section{Coupled Hindmarsh-Rose Model System}

. In the nervous system, according to the structure and transmission mechanism of synapses, it can be divided into chemical synapses and electrical synapses. In this paper, the synapse is a system coupled with two neurons. Based on the modified HR model, the electrical and chemical synapse model under TMAES was established. Assume that the pre-synaptic and post-synaptic neuron pairs have the same effect on each other. TMAES is applied to presynaptic neurons.

The expression of the two HR neuron models of coupled excitatory-excitatory synapse under TMAES is described by (9), where $k_{1}$ is the synaptic coupling strength and $k_{2}$ is the electric field coupling strength. $k_{1}$ and $k_{2}$ are both set to 0.5. $u$ is an environmental variable, represents the positive feedback of mutually coupled neurons is an indirect coupling [25][27]. To show the influence of presynaptic neurons on postsynaptic neurons, make $G_{1}=u$. $G_{2}$ means other external effects except for TMAES, considering that neuroelectric signals can only be transmitted from presynaptic neurons to postsynaptic neurons. In the chemical synapse, $G_{1}=u, G_{2}=0$, and for the electrical synapse $G_{2}=G_{1}=u[27]$.

$$
\left\{\begin{aligned}
C_{m} \frac{d x_{1}}{d t} & =y_{1}-x_{1}^{3}+3 x_{1}^{2}-z_{1}+I_{e x t}-k_{1}\left(x_{1}^{3}-x_{2}^{3}\right)+G_{1} \\
\frac{d y_{1}}{d t} & =1-5 x_{1}^{2}-y_{1}+r E_{1} \\
\frac{d z_{1}}{d t} & =0.006\left[4\left(x_{1}+1.56\right)-z_{1}\right] \\
\frac{d E_{1}}{d t} & =k y_{1}+\boldsymbol{E}_{e x t}+k_{2}\left(E_{2}-E_{1}\right) \\
\frac{d C_{m}}{d t} & =(2 \pi f) C_{a m p} \cos (2 \pi f t) \\
C_{m 0} \frac{d x_{2}}{d t} & =y_{2}-x_{2}^{3}+3 x_{2}^{2}-z_{2}-k_{1}\left(x_{2}^{3}-x_{1}^{3}\right)+G_{2} \\
\frac{d y_{2}}{d t} & =1-5 x_{2}^{2}-y_{2}+r E_{2} \\
\frac{d z_{2}}{d t} & =0.006\left[4\left(x_{2}+1.56\right)-z_{2}\right] \\
\frac{d E_{2}}{d t} & =k y_{2}+k_{2}\left(E_{1}-E_{2}\right) \\
\frac{d u}{d t} & =-0.1 u-\frac{1}{2}\left(x_{1}+x_{2}\right)
\end{aligned}\right.
$$$$
\text { (9) }
$$

\section{TMAES waveform}

The electric field induced by TMAES is the product of the coupling of ultrasound and static magnetic fields. We used modulated ultrasound waves to simulate TMAES. In this paper, the ultrasonic frequency $(f)$ is $690 \mathrm{kHz}$, which is modulated by a square wave with an intensity $A$, a frequency $f_{s q}$, and a duty cycle $D$. The TMAES consists of a periodic modulated sine wave. The sine wave with frequency $f$ represents the unmodulated ultrasound, and the square wave with frequency $f_{s q}$ is the modulated wave.

Figure 2 shows the TMAES waveform, where is the square wave modulating sinusoidal ultrasonic wave with frequency 
$690 \mathrm{kHz}$. The modulated ultrasound is used for TMAES to study the effect of TMAES on coupled two neurons firing activity. In this paper, the modulated ultrasound was used as TMAES to act on the simulation of the influence of TMAES on the coupled Hindmarsh-Rose model. the parameters of the square wave were changed to alter the TMAES. TMAES parameters: modulated frequency $f_{s q}$, duty cycle $(D)$, and intensity $A$.

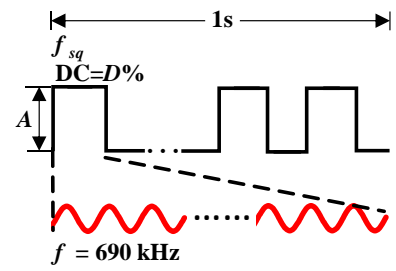

Figure 2. TMAES waveform

\section{RESULTS}

Electrical and chemical synapse were simulated, respectively, under the TMAES with different intensities during the first one second. In this paper, we consider the effects of the two parameters, which are the intensity $A$ and the modulated frequency $f_{s q}$.

\section{A. Model(1): electrical synapse}

TMAES with modulation frequency $f_{s q}$ and duty cycle $D$ were $50 \mathrm{~Hz}$ and 50 . TMAES intensity $A$ is $0.1,0.2,0.3$ and 0.5 , respectively. The time series of two neurons' membrane potential obtained is shown in Fig. 3. X1 represents the membrane potential of the presynaptic neuron, and $\mathrm{X} 2$ represents the membrane potential of the postsynaptic neuron. Short-term stimulation $(<250 \mathrm{~ms})$ has a higher neuron firing frequency and a stronger synchronization of neurons than long-term stimulation $(>250 \mathrm{~ms})$ are shown in Fig. 3. And the nervous system's firing appears as an asynchronous firing. Long-term stimulation reduces the firing frequency. A postsynaptic neuron is bursting during long-term stimulation. As the TMAES intensity increases, the amplitude increases slightly under the short-term stimulation as the intensity increases, and the long-term stimulation decreases membrane potential amplitude of bursting. When $A$ was 0.5 , the early synchronization stage is shortest and the later bursting stage is the longest in Fig. 3. When the intensity $A$ is $0.1,0.2,0.3$, respectively, the firing frequency of the model increases as the TMAES intensity increases. When $A$ was $0.2,0.3,0.5$, respectively, the membrane potential amplitude exhibit a slight increase, the electrical synapse discharge regularity decreases in the bursting stage. This shows that only stimulation within a certain intensity range can make neurons into a periodic discharge state. When the stimulation intensity is different, the stimulation duration affects the neuron firing activity and then affects the synchronization duration of the electrically coupled neurons system. Besides the electrical coupling system is selective to the stimulation intensity.

To know how the TMAES modulation frequency $f_{s q}$ acts on the firing of the electrical synapse, the intensity $A$, and duty cycle $D$ were 0.5 and 50 . The modulation frequency is $25 \mathrm{~Hz}$, $50 \mathrm{~Hz}, 75 \mathrm{~Hz}$, and $100 \mathrm{~Hz}$, respectively. The coupled neurons' firing activities under different modulation frequencies are shown in Fig. 4. The synaptic firing frequency of the electrical synapse is affected by the modulation frequency. With the modulation frequency increasing, the neuron firing frequency also increases, and the early spiking time decreases at first and then increases. The $f_{s q}$ also affects the synapse firing frequency. In addition, as the $f_{s q}$ increases, TMAES changes rapidly causing frequent dielectric polarizing. Under different $f_{s q}$ of TMAES, the short-term stimulation amplitude varies very little, and membrane potential amplitude during the long-term stimulation becomes smaller. The electrical coupling system is selective to the modulation frequency. According to the required stimulation effect, the appropriate modulation frequency can be selected.
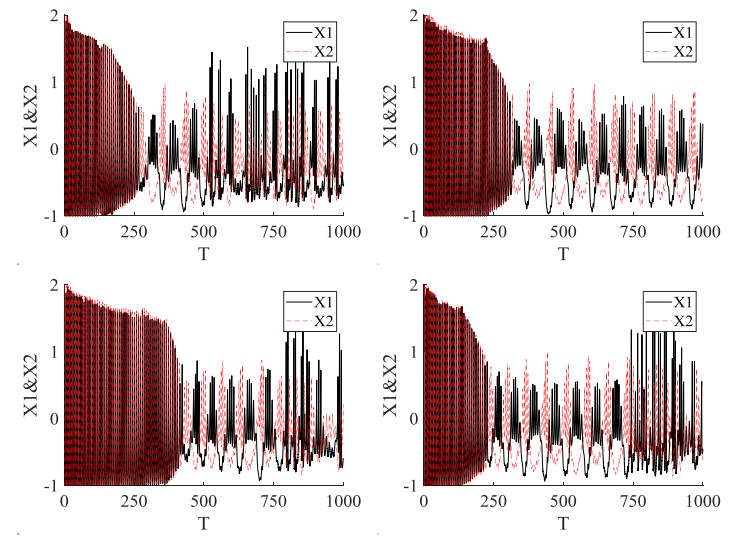

Figure 3. TMAES with different intensities on the electrical synapse, $A=0.1,0.2,0.3,0.5$, respectively. Initial values for all the figures are $[1,1,1,1,1,1,1,1,1,1]$.
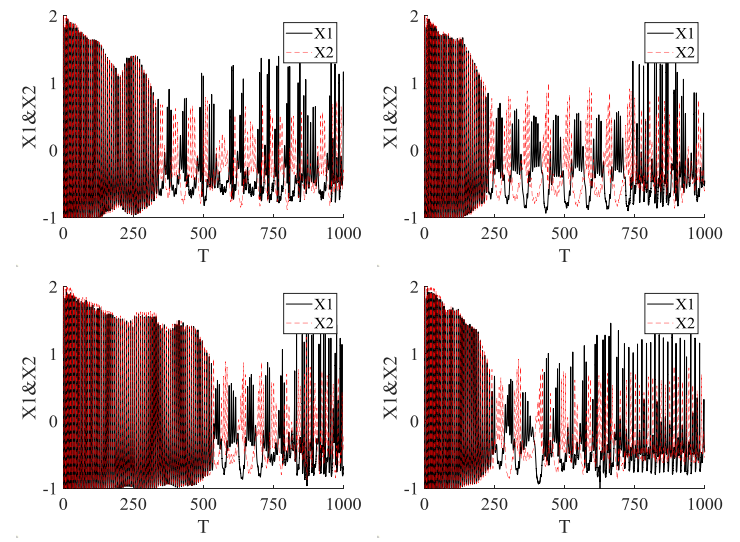

Figure 4. TMAES with different modulation frequency $f_{s q}$ on the electrical synapse, $A=0.5, D=50$ respectively. Initial values for all the figures are $[1,1,1,1,1,1,1,1,1,1]$.

\section{B. Model(2): chemical synapse}

Different firing behaviors can be observed by changing the TMAES intensity. The TMAES intensity should be set to be the same as the intensity in Model (1), but the postsynaptic neuron has no action potential as $A=0.1$, so the stimulation intensity $A$ is 
$0.1,1,3$, and 5 , respectively. TMAES modulation frequency $f_{s q}$ and duty cycle $D$ were $50 \mathrm{~Hz}$ and 50 . TMAES intensity $A$ is 0.1 , $1,3,5$, respectively. The two neurons coupling chemical synaptic discharge activity under different intensity is shown in Fig. 5. The presynaptic neuron X1 receives TMAES directly. The first action potential of the postsynaptic neuron X2 firing after $500 \mathrm{~ms}$ as $A=3$ and the periodic action potential of the postsynaptic neuron appeared at $250 \mathrm{~ms}$ as $A$ is 5 . With the increase of stimulation intensity, the earlier the periodic postsynaptic action potential appeared. It can be observed that the TMAES accelerated the firing of the first action potential of postsynaptic neurons. Unlike electrical synapses, chemical synapses are not synchronized, the speed of the information transmission of the chemical synapse is slower than the electrical synapse. It's caused by the difference in the information transmission mechanism of the chemical synapse is more complicated than the electrical synapses. The greater the stimulation intensity, the lag time between the action
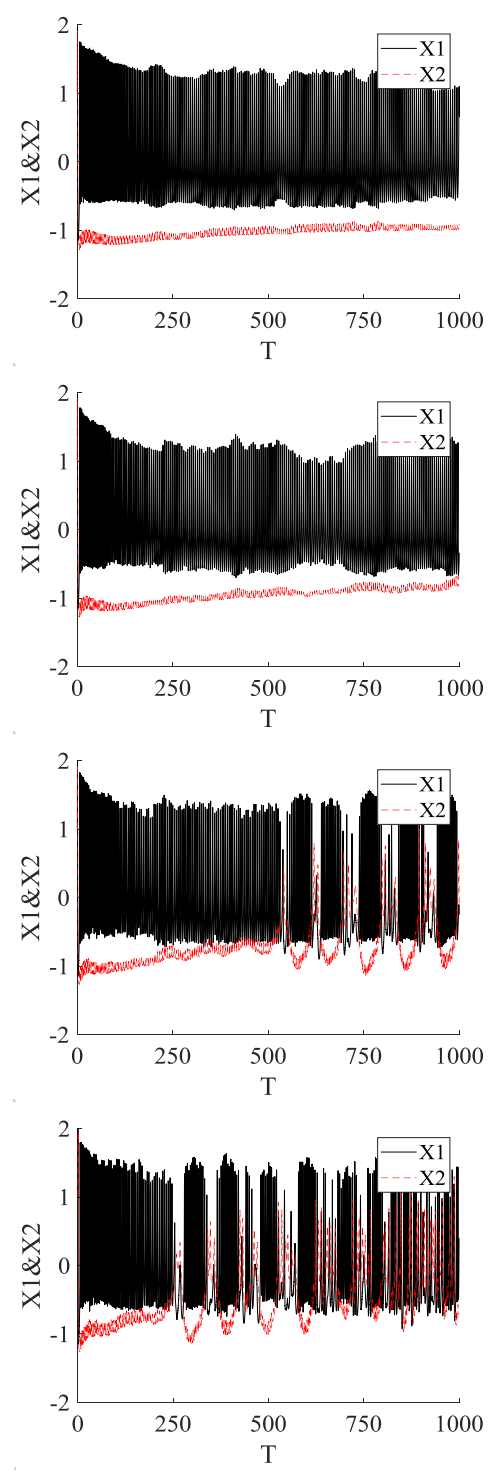

Figure 5. TMAES with different intensities on the chemical synapse, $A=0.1,1,3,5$, respectively. Initial values for all the figures are $[1,1,1,1,1,1,1,1,1,1]$

potentials of the presynaptic and postsynaptic neurons of the chemical synapse. And it may be because TMAES accelerate the information processing of the coupled neural system.

With the increase of stimulation intensity, the firing frequency of postsynaptic neurons increases. Compared with electrical synapse, exciting postsynaptic neuron requires bigger TMAES intensity. TMAES has no significant effect on the amplitude of neuronal action potentials between synapse-coupled neurons. Increasing the TMAES intensity can increase the firing rate of neurons. It mainly adjusts the firing frequency of the neural system. This may be due to the redistribution of ions caused by the induced electric field and the change of ion concentration. The nerve excitability is increased by the regulatory effect on the neuronal electrical activity of TMAES.

The intensity A is $5, D$ is 50 , only the modulation frequency 
$f_{s q}$ is changed. The excitation-excitation coupled chemical
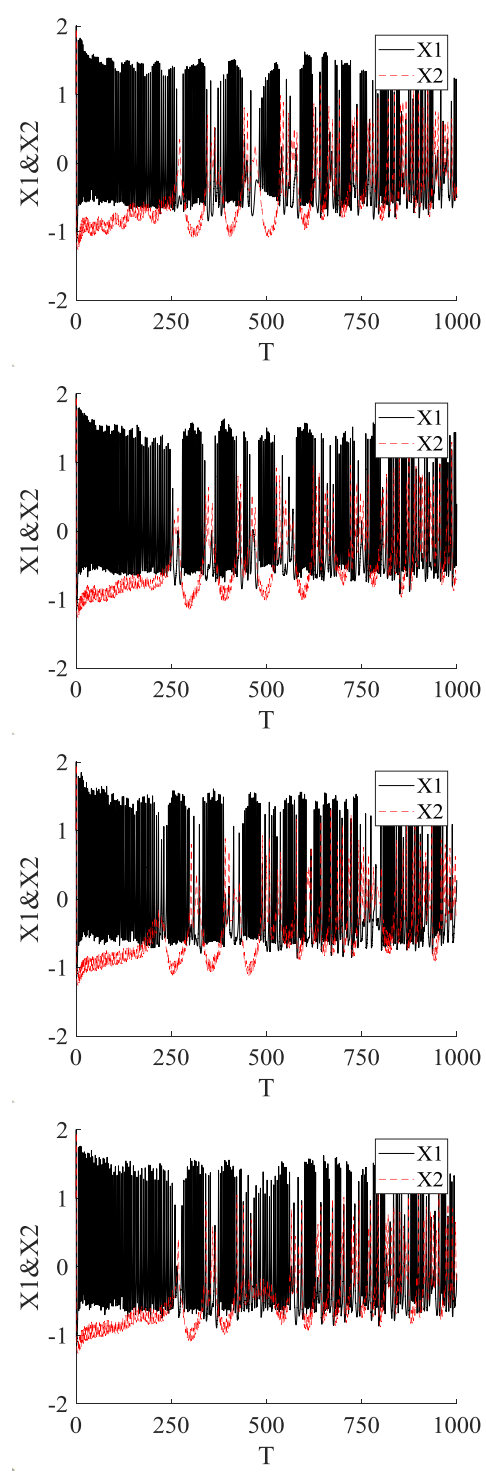

Figure 6. TMAES with different modulation frequency $f_{s q}$ on the chemical synapse, $A=5, D=50$ respectively. Initial values for all the figures are $[1,1,1,1,1,1,1,1,1,1]$.

synaptic neuron membrane potential obtained at different modulation frequencies $f_{s q}$ is shown in Fig. 6. Short-term stimulation produces faster first action potentials of presynaptic neurons. The firing activity of neurons in this coupling system is not synchronized. In addition, as the modulation frequency increases, the first action potential of a postsynaptic neuron appears earlier. The discharge of the chemical synapse appears as an asynchronous discharge. The chemical coupling system is selective to the modulation frequency of the TMAES.

\section{CONCLUSION}

TMAES is a new type of non-invasive neural control technology with high spatial resolution. The coupled electric field is generated by ultrasound and static magnetic field, which indirectly generates stimulation in nerve tissue. Changing the parameters of TMAES can regulate neuron excitability and affect the transmission of information between neurons in the cerebral cortex. It suggests its potential for achieving the purpose of treating neuropsychiatric diseases and contributing to the prevention and treatment of brain diseases. They may elucidate new physical cellular effects of nerve regulation and may lead to potential advanced methods of nerve control.

It provides references for the intervention and treatment of neuropsychiatric diseases caused by abnormal neuronal firing rhythm, provides theoretical support for the treatment of neuropsychiatric diseases by TMAES, and contributes to studying the potential mechanism of information processing in the nervous system.

In the next step, we will establish a corresponding experimental system to verify the regulatory effect of transcranial magnetic acoustic stimulation on neurons and the nervous system and promote the research of the clinical application of TMAES.

\section{ACKNOWLEDGMENT}

This work was supported by the National Natural Science Foundation of China under Grant No.51877069.

\section{References}

[1] E Gianni, M Bertoli, L Simonelli, et al. "tDCS randomized controlled trials in no-structural diseases: a quantitative review," Scientific reports, vol. 11, no.1, pp. 16311-16311, Aug. 2021.

[2] F Thomas, F Steinberg, NH Pixa, et al. "Prefrontal high definition cathodal tDCS modulates executive functions only when coupled with moderate aerobic exercise in healthy persons," Scientific reports, vol. 11, no.1, pp. 8457, Apr. 2021.

[3] S Lerma-Lara, MD Montbron, $M$ Guerin, et al. "Transcranial direct-current stimulation (tDCS) in the primary motor cortex and its effects on sensorimotor function: a quasi-experimental single-blind sham-controlled trial," Scientific reports, vol. 11, no.1, pp. 6566, Mar. 2021.

[4] E. Kaminski, M. Engelhardt, M. Hoff, et al. "TDCS effects on pointing task learning in young and old adults," Scientific reports, vol. 11, no.1, pp. 3421, Feb. 2021.

[5] K. Heimrath, A. Brechmann, R. Blobel-Lüer, et al. "Transcranial direct current stimulation (tDCS) over the auditory cortex modulates GABA and glutamate: a $7 \mathrm{~T}$ MR-spectroscopy study," Scientific reports, vol. 10, no.1, pp. 20111, Nov. 2020.

[6] Y Xia, W Khalid, Z Yin, et al. "Modulation of solute diffusivity in brain tissue as a novel mechanism of transcranial direct current stimulation (tDCS)," Scientific reports, vol. 10, no.1, pp. 18488, Oct. 2020.

[7] Y Xia, W Khalid, Z Yin, et al. "Low-Frequency Transcranial Magnetic Stimulation (LF-TMS) in Treating Depression in Patients With Impaired Cognitive Functioning," Archives of Clinical Neuropsychology, vol. 36, no.5, pp.801-814, Jul. 2021.

[8] JH Deng, WM Fang, YM Gong, et al. "Augmentation of fear extinction by theta-burst transcranial magnetic 
stimulation of the prefrontal cortex in humans," Journal of psychiatry and neuroscience, vol. 46, no.2, pp. E292-E302, Mar. 2020.

[9] A M Fukuda, L E Hindley, J W D Kang, et al. "Peripheral vascular endothelial growth factor changes after transcranial magnetic stimulation in treatment-resistant depression," NeuroReport, vol. 31, no.16, pp. 1121-1127, Nov. 2020.

[10]S Balestrini, JW Sander. "Transcranial magnetic stimulation as a biomarker of treatment response in children with epilepsy," Developmental Medicine and Child Neurology, vol. 62, no.7, pp. 793-798, Jul.2020.

[11]B Clennell, TGJ Steward, M Elley, et al. "Transient ultrasound stimulation has lasting effects on neuronal excitability," Brain Stimulation, vol. 14, no. 2, pp. 217-225, Mar. 2021.

[12] S Kim, Y Jo, G Kook, et al. "Transcranial focused ultrasound stimulation with high spatial resolution," Brain Stimulation, vol. 14, no. 2, pp. 290-300, Mar. 2021.

[13] JX Wang, GF Li, LD Deng, et al. "Transcranial Focused Ultrasound Stimulation Improves Neurorehabilitation after Middle Cerebral Artery Occlusion in Mice," Aging and disease, vol. 12, no. 1, pp. 50-60, Feb. 2021.

[14]N Pang, XW Huang, H Zhou, et al. "Transcranial Ultrasound Stimulation of Hypothalamus in Aging Mice," IEEE transactions on ultrasonics, ferroelectrics, and frequency control, vol. 68, no. 1, pp. 29-37, Jan. 2021.

[15] K Hynynen, "Demonstration of enhanced temperature elevation due to nonlinear propagation of focused ultrasound in dog's thigh in vivo," Ultrasound in Medicine and Biology, vol. 13, no. 2, pp.85-91, Feb. 1987.

[16] W L Nyborg, "Biological effects of ultrasound: development of safety guidelines. Part II: general review," Ultrasound in Medicine and Biology, vol. 27, no. 3, pp. 301-333, Mar. 2001.

[17]XQ Zhou, SK Liu, YX Wang, et al. "High-Resolution Transcranial Electrical Simulation for Living Mice Based on Magneto-Acoustic Effect," Frontiers in Neuroscience, vol. 13, no. 1342, pp. 1-12, Dec, 2019.

[18] S.J. Norton, "Can ultrasound be used to stimulate nerve tissue?" Advanced Biomedical Engineering, vol. 2, no. 1, pp. 6, Mar. 2003.

[19] Y Yuan and X Li, "Theoretical analysis of transcranial Hall-effect stimulation based on passive cable model," Chinese Physics B, vol. 24, no. 12, pp. 373-378, Dec. 2015.

[20]E Izhikevich, "Which model to use for cortical spiking neurons?" IEEE Transactions on Neural Networks, vol. 15, no. 5, pp. 1063-1070, Sep. 2004.

[21] S Zhang, K Cui, and X Zhang, et al., "Effect of Transcranial Ultrasonic-Magnetic Stimulation on Two Types of Neural Firing Behaviors in Modified Izhikevich Model," IEEE Transactions on Magnetics, vol. 54, no. 3, pp.1-4, Feb. 2018.

[22] A. L. Hodgkin and A. F. Huxley, "A quantitative description of membrane current and its application to conduction and excitation in nerve," Japanese Journal of Physiology, vol. 117, no. 4, pp. 500-544, Aug. 1952.
[23] J L Hindmarsh and R M Rose, "A model of the nerve impulse using two first-order differential equations," Nature, vol. 296, no. 5853, pp. 162-164, Mar. 1982.

[24] J L Hindmarsh and R M Rose, "A model of neural bursting using three coupled first order differential equations," Proceedings of the Royal Society of London. Series B, Biological sciences, vol. 221, no. 1222, pp. 87-102, Mar. 1984.

[25] S.K. Thottil, and R.P. Ignatius, "Nonlinear feedback coupling in Hindmarsh-Rose neurons," Nonlinear Dynamics, vol. 87, no. 3, pp. 1879-1899, Feb. 2017.

[26] Usha Kaniyattil, and P.A. Subha, "Collective dynamics and energy aspects of star-coupled Hindmarsh-Rose neuron model with electrical, chemical and field couplings," Nonlinear Dynamics, vol. 94, no. 4, pp. 2115-2124, May. 2019.

[27]S Mostaghimi, F Nazarimehr, S Jafari, and J Ma, "Chemical and electrical synapse-modulated dynamical properties of coupled neurons under magnetic flow," Applied Mathematics and Computation, vol. 348, pp. 42-56, May. 2019.

[28] J Ma, G Zhang, T Hayat, and G Ren, "Model electrical activity of neuron under electric field," Nonlinear Dynamics, vol. 95, no. 2, pp. 1585-1598, Jan. 2018.

[29]R. Fitzhugh, "Thresholds and Plateaus in the Hodgkin-Huxley Nerve Equations," The Journal of General Physiology, vol. 43, no. 5, pp. 867-896, May. 1960.

[30] HY Chen, A Jerusalem, “A Framework for Low-Intensity Low-Frequency Ultrasound Neuromodulation Sonication Parameter Identification from Micromechanical Flexoelectricity Modelling," Ultrasound in medicine and biology, vol. 47, no.7, pp:1985-1991, Jul. 2021.

[31] T Lemaire, E Neufeld, and N Kuster, et al., "Understanding ultrasound neuromodulation using a computationally efficient and interpretable model of intramembrane cavitation," Journal of neural engineering vol. 16, no. 4, pp. 046007-046025, Aug. 2019.

[32] M Plaksin, E Kimmel, S Shoham, "Cell-Type-Selective Effects of Intramembrane Cavitation as a Unifying Theoretical Framework for Ultrasonic Neuromodulation," eNeuro, vol. 3, no, 3, pp. 1-16, May. 2016.

[33]EA Rashed, J Gomez-Tames, and A Hirata, "Deep Learning-Based Development of Personalized Human Head Model with Non-Uniform Conductivity for Brain Stimulation," IEEE transactions on medical imaging, vol. 39, no. 7, pp. 2351-2362. Jul. 2020. 
Contribution of Individual Authors to the Creation of a Scientific Article (Ghostwriting Policy)

Liang Guo wrote this paper carried out the simulation and the optimization; Shuai Zhang revised the paper; Xinyu Gao drew the schematic in this paper; Jiankang $\mathrm{Wu}$ reviewed and edited it; Mingkang Zhao proposed some suggestions; Guizhi Xu was responsible for supervision. All authors discussed the results and wrote the manuscript and gave final approval for publication.

http://naun.org/main/format/contributor-role.pdf
Creative Commons Attribution License 4.0 (Attribution 4.0 International, CC BY 4.0)

This article is published under the terms of the Creative Commons Attribution License 4.0

https://creativecommons.org/licenses/by/4.0/deed.en_US 